\title{
Les techniques numériques et l'histoire des techniques. Le cas des maquettes virtuelles animées
}

Study of the virtual dynamic scale models

\section{Michel Cotte}

\section{(2) OpenEdition Journals}

Édition électronique

URL : http://journals.openedition.org/dht/97

DOI : $10.4000 /$ dht. 97

ISSN : 1775-4194

Éditeur :

Centre d'histoire des techniques et de l'environnement du Cnam (CDHTE-Cnam), Société des élèves du CDHTE-Cnam

Édition imprimée

Date de publication : 1 décembre 2009

Pagination : 7-21

ISBN : 978-2-9530779-4-0

ISSN : 0417-8726

\section{Référence électronique}

Michel Cotte, «Les techniques numériques et l'histoire des techniques. Le cas des maquettes virtuelles animées », Documents pour l'histoire des techniques [En ligne], $18 \mid 2^{\mathrm{e}}$ semestre 2009, mis en ligne le 24 septembre 2010, consulté le 08 septembre 2020. URL : http://journals.openedition.org/dht/ 97 ; DOI : https://doi.org/10.4000/dht.97 


\title{
Les techniques numériques et l'histoire des techniques. Le cas des maquettes virtuelles animées
}

\author{
Michel Cotte \\ Centre François Viète d'histoire des sciences et des techniques \\ Université de Nantes / Icomos / unité du patrimoine mondial Unesco
}

\begin{abstract}
RÉSUMÉ
Le présent numéro des Documents pour l'histoire des techniques est consacré à la question des techniques numériques comme outils possibles de l'histoire des sciences et des techniques, ainsi que du patrimoine industriel et de la muséographie des techniques. Depuis le début des années 1990, des avancées importantes et rapides ont été effectuées dans plusieurs directions : la numérisation des documents, la production de dessins assistés par ordinateur, les photos numériques, les bases de données, internet, etc. Quelques historiens des techniques ont également envisagé l'application d'outils plus spécialisés, mis au point par d'autres communautés, comme les maquettes tridimensionnelles des architectes, la CAO (conception assisté par ordinateur) des sciences pour l'ingénieur, ou les SIG (systèmes d'informations géographiques) des géographes et des urbanistes. Des usages croisés entre ces différentes techniques numériques font aujourd'hui partie des recherches en cours, à propos notamment de l'histoire des techniques. Ce numéro des DHT dresse une image de ces nouvelles possibilités de travail dans nos disciplines, à partir d'expériences menées par différents collègues au sein de différentes institutions. Cet ensemble n'est pas exhaustif, mais il souhaite montrer le dynamisme actuel des usages du numérique dans nos disciplines, le faire partager, enfin ouvrir un débat tant sur les perspectives que sur les nombreuses questions posées par ces nouveaux outils.
\end{abstract}

Résumés et mots-clés en anglais sont regroupés en fin de volume, accompagnés des mots-clés français

es techniques à base numérique n'ont a priori pas

- grand-chose à voir avec les disciplines de l'histoire, même si par nature elles appartiennent au champ de l'histoire des sciences et des techniques, celle-ci se devant de décrire leurs évolutions et leurs usages au fil du temps. Ce n'est toutefois pas de cela dont il est question dans cette livraison des Documents pour l'histoire des techniques, mais plutôt de l'impact de ces techniques sur notre manière de faire de l'histoire comme d'en présenter les résultats. Nous nous préoccupons ici de leur utilisation possible en tant qu' outils éventuels de l'histoire des techniques et des sciences, plus largement du patrimoine industriel et de la muséographie des techniques. II s'agit d'en saisir l'intérêt, la portée, de préciser les modes d'emplois, mais aussi d'en cerner les limites comme les interrogations épistémologiques auxquelles elles peuvent parfois conduire.
Les technologies numériques ont complètement bouleversé la plupart des champs professionnels, non seulement dans la transmission et la gestion de l'information, mais aussi, souvent, dans la manière de travailler. C'est un lieu commun de le constater. Par les nouvelles possibilités d'action offertes par les logiciels, les méthodes de travail ont rapidement évolvé, parfois les données mêmes de la connaissance ont franchi des sevils significatifs. Des capacités d'analyse, d'évaluation ou de comparaison jusque-là inenvisageables ont été mises en œuvre. Le traitement de l'information s'est considérablement étendu, et ces technologies offrent des approches de recherche comme de présentation des résultats entièrement nouvelles. Nul champ professionnel ou scientifique ne peut échapper à leur usage, sous une forme ou sous une autre, nul ne peut sérieusement envisager de ne 
pas y réfléchir dans une perspective d'évolution de sa propre discipline et la manière renouvelée d'en produire les connaissances. C'est ce qu'envisage ce numéro de DHT, comme un premier bilan de résultats confirmés, d'essais pratiques, de recherches et d'échanges ayant eu lieu depuis près de dix ans. Nous ne pouvons pas faire l'économie de la " révolution du numérique », aujourd'hui, ni éviter de s'interroger de manière critique sur les conséquences de cette rupture radicale intervenue dans le système sociotechnique contemporain.

\section{Les techniques numériques et l'histoire des techniques} Les éléments de notre travail pouvant être concernés par les nouveaux outils du numérique sont nombreux. Nous pouvons les présenter assez simplement, sous forme d'un tableau où nous plaçons en colonnes l'objet de notre étude et les champs dans lesquels il s'inscrit : historique, archéologique, patrimoine industriel, muséographie, etc. ; en lignes horizontales, nous mettons les outils numériques existants ou en développement : procédés de numérisation, bases de données, internet et sites web, conception assistée par ordinateur (CAO), etc. Chaque case du tableau indique I'utilisation possible d'un outil numérique. Le tableau forme une grille récapitulative des traitements numériques envisageables à propos d'un objet technique donné. Un projet concret peut se ramener à une case, plus généralement à plusieurs qui s'enchaînent. Il est bien entendu possible d'ajouter des lignes comme des colonnes au tableau, c'est-àdire de nouveaux outils et de nouveaux domaines d'application.

Prenons un exemple : l'étude de la machine à vapeur Piguet de l'Écomusée du Creusot par les étudiants de l'Université de Technologie de BelfortMontbéliard, au cours des années 2000-2003'. Cette

1 Michel Cotte et Samuel Deniaud, "Conception assistée par ordinateur et patrimoine, perspectives innovantes $"$, L'archéologie industrielle en France, 46, juin 2005, pp. 32-38.

Exemple d'une « matrice de projets » impliquant diverses techniques numériques

\begin{tabular}{|c|c|c|c|c|}
\hline $\begin{array}{l}\text { Machine à vapeur } \\
\text { Piguet du Creusot }\end{array}$ & $\begin{array}{l}\text { Éłude historique, } \\
\text { documentation }\end{array}$ & $\begin{array}{c}\text { Étude archéologique } \\
\text { des vestiges }\end{array}$ & $\begin{array}{l}\text { Projet } \\
\text { patrimonial }\end{array}$ & $\begin{array}{c}\text { Projet } \\
\text { muséographique }\end{array}$ \\
\hline $\begin{array}{l}\text { Numérisation des } \\
\text { données }\end{array}$ & Photos numériques & $\begin{array}{l}\text { Numérisation 3D des } \\
\text { pièces, } \\
\text { Nuages de points }\end{array}$ & $\begin{array}{l}\text { Illustrations sur } \\
\text { site, compléments } \\
\text { d'information }\end{array}$ & $\begin{array}{l}\text { Documentation } \\
\text { complémentaire à } \\
\text { l'objet matériel }\end{array}$ \\
\hline $\begin{array}{l}\text { DAO (dessin } \\
\text { assisté par } \\
\text { ordinateur) }\end{array}$ & & Plans numériques & \multicolumn{2}{|c|}{ Plans 2D restitués de la machine } \\
\hline $\begin{array}{l}\text { CAO } \\
\text { (conception } \\
\text { assistée par } \\
\text { ordinateur) }\end{array}$ & \multicolumn{2}{|c|}{ Étude mécanique et cinématique } & $\begin{array}{l}\text { Maquette numérique } \\
\text { dynamique, dossier } \\
\text { d'œuvre numérique }\end{array}$ & $\begin{array}{c}\text { Maquette numérique } \\
\text { dynamique, } \\
\text { complément } \\
\text { d'information }\end{array}$ \\
\hline Bases de données & $\begin{array}{l}\text { Documentation sur } \\
\text { l'histoire de la machine } \\
\text { histoire économique } \\
\text { et sociale }\end{array}$ & $\begin{array}{l}\text { Documentation sur } \\
\text { l'environnement de } \\
\text { la machine }\end{array}$ & \multicolumn{2}{|c|}{$\begin{array}{l}\text { Inscription des résultats dans les bases de } \\
\text { données locales, nationales, internationales... } \\
\text { Lien maquette numérique - base de données }\end{array}$} \\
\hline Film numérique & & & \multicolumn{2}{|c|}{ Présentation pédagogique de la maquette } \\
\hline Interactivité & & & \multicolumn{2}{|c|}{$\begin{array}{c}\text { Développements pédagogiques en } \\
\text { fonction des objectifs du projet et des } \\
\text { moyens logiciels }\end{array}$} \\
\hline $\begin{array}{l}\text { Vision } \\
\text { stéréoscopique }\end{array}$ & & & \multicolumn{2}{|c|}{$\begin{array}{c}\text { Installation spécialisée en version grand } \\
\text { public, lieu dédié }\end{array}$} \\
\hline $\begin{array}{l}\text { Restitution des } \\
\text { ambiances, des } \\
\text { processus... }\end{array}$ & $\begin{array}{l}\text { Acquisition } \\
\text { de données } \\
\text { supplémentaires }\end{array}$ & $\begin{array}{l}\text { Acquisition } \\
\text { de données } \\
\text { supplémentaires }\end{array}$ & \multicolumn{2}{|c|}{$\begin{array}{c}\text { Développements en fonction des objectifs } \\
\text { du projet et des moyens logiciels }\end{array}$} \\
\hline $\begin{array}{l}\text { Site web, accès à } \\
\text { distance }\end{array}$ & & & $\begin{array}{c}\text { Page web sur le } \\
\text { patrimoine, liens, } \\
\text { sites collectifs et de } \\
\text { réseaux }\end{array}$ & $\begin{array}{l}\text { Page web du } \\
\text { musée, liens, sites } \\
\text { collectifs et de } \\
\text { réseaux }\end{array}$ \\
\hline
\end{tabular}


étude a concerné plusieurs champs disciplinaires, à différents moments, par plusieurs étudiants; mais tous les projets envisageables n'ont pas forcément été réalisés; certains ne s'inscrivaient pas dans une perspective numérique, qui n'est finalement intervenue que dans un second temps, lorsque le traitement conventionnel d'une machine vestige est arrivé à ses limites ${ }^{2}$. Nous complétons la grille par des aspects envisagés à propos d'autres études, afin de rendre compte de la diversité possible des cases du tableau ou matrice de projets. C'est donc un exemple idéalisé où tout n'a pas été réalisé. Toutefois, en pratique, cette étude a permis de reconstituer virtuellement la machine et de pleinement comprendre son fonctionnement à partir d'éléments mécaniques démontés et disparates. Par ailleurs, et c'est une remarque importante, le numérique ne remplace pas une étude classique de l'objet, mais il la complète. II s'inscrit dans un projet historique ou patrimonial bien plus vaste et au service duquel il se met. II s'agit d'un outil d'analyse et de présentation des résultats qui ne remplace pas les méthodes habituelles de l'histoire ou du patrimoine, mais qui vient leur ajouter des possibilités nouvelles.

Les maquettes numériques concrétisent la connaissance matérielle d'un objet technique, surtout Iorsqu'elles sont capables d'apporter la dimension cinématique, c'est-à-dire de restituer les mouvements mécaniques des pièces entre elles au cours du temps. Elles sont l'un des points d'entrée de ce numéro de DHT, et un sujet d'approfondissement car il a focalisé une part importante et originale des efforts en histoire des techniques, avec notamment les articles de Florent Laroche sur les concepts de capitalisation des connaissances du passés par les outils d'aujourd'hui : "Une nouvelle forme de capitalisation des connaissances grâce à l'archéologie industrielle avancée ", de Sylvain Laubé sur la mise en perspective des expériences de maquettes numériques animées menées par l'équipe de Brest : "Modélisation des documents numériques pour l'histoire des techniques: une perspective de recherche "I, ou l'article de Stéphane Sire, Dominique Cochou et Jean-François Péron sur l'usage de ces outils dans un projet de restauration-conservation d'un patrimoine : « De l'aide des maquettes virtuelles à la restauration d'un ouvrage d'art historique, le viaduc de Lambézellec (Brest) ॥. Nous donnons nous même un essai de mise en perspective de l'expérience

2 Nous avons tout d'abord envisagé de remonter la machine réelle, ce qui s'est avéré quasiment impossible avec les moyens à notre disposition ; par ailleurs des pièces importantes manquaient comme la chaudière. acquise à propos des maquettes numériques de machines dans la suite de cet article introductif.

L'usage stricto sensu de la CAO correspond à l'une des cases du tableau seulement; ce n'est qu'un domaine bien spécialisé du numérique parmi d'autres. Toutefois, il entretien des liens avec les autres domaines dans le développement des projets, notamment les bases de données qui ont rapidement été à l'ordre du jour de notre groupe, rejoignant les préoccupations d'autres équipes. II s'agit notamment de l'expérimentation d'Alain Michel dans sa description-compilation d'un atelier : «La reconstitution virtuelle d'un atelier de RenaultBillancourt : sources, méthodologie et perspectives 1.

Dans d'autres champs des techniques numériques, des projets précis au bénéfice des historiens dessciences et des techniques peuvent prendre une très grande extension, comme la numérisation documentaire des livres anciens, enjeu aujourd'hui d'importance internationale. Nous savons que ces possibilités nouvelles ont en quelques années totalement modifié les conditions d'étude des documents originaux ou rares, en permettant de les consulter chez soi. Dans ce domaine, nous avons le plaisir d'accueillir l'article collectif du Conservatoire numérique des arts et métiers (Cnum) : «Le Conservatoire numérique : enjeux et perspectives de la numérisation documentaire $\|^{3}$. Il fait le point sur les résultats obtenus, à partir des collections exceptionnelles du Conservatoire des arts et métiers, et sur la pratique de la numérisation au profit de l'histoire des sciences et des techniques. Dans le domaine de la constitution des bases de données et de leur usage interactif à distance, par le web, le projet de compilation du patrimoine scientifique universitaire est aujourd'hui en cours de réalisation, à l'échelle de la France, après une première expérience réussie à Nantes et en Pays de la Loire. L'article d'Yves Thomas et Catherine Cuenca, les promoteurs et les animateurs de ce projet, donne un compterendu de cette expérience et de ses perspectives ${ }^{4}$ : "L'apport des technologies de l'information et de la communication (TIC) à la sauvegarde du patrimoine scientifique et technique contemporain $\%$. Dans un domaine plus expérimental à ce jour, celvi des systèmes d'informations géographiques (SIG) appliqués au patrimoine, Jean-Louis Kérovanton présente un projet en cours sur le port de Nantes : «Pour l'utilisation des SIG (systèmes d'information géographique) en histoire des techniques: entre documentation et analyse spatiale $»$.

\footnotetext{
3 cnum.cnam.fr/

4 patrimoine.atlantech.fr/
} 


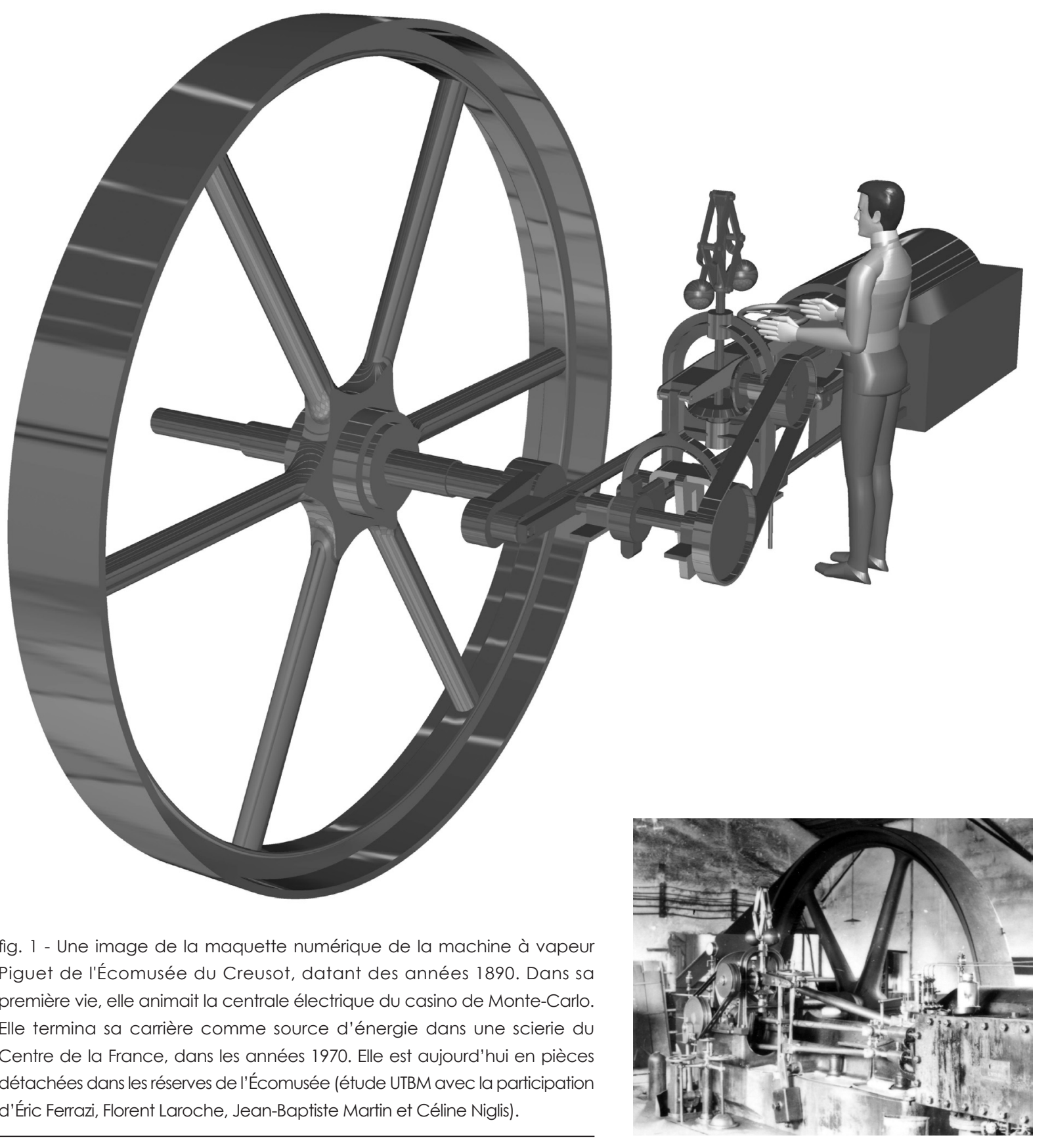

Par ces réalisations, nous voyons la tendance fréquente à associer plusieurs techniques numériques, à avoir des projets évoluant sur plusieurs cases du tableau. II y a notamment la recherche d'une interactivité évolvée avec les utilisateurs, qui intéresse particulièrement les muséographes. II est essentiel de pouvoir établir des liens, de faire des comparaisons, d'envisager des complémentarités entre, par exemple, les outils de visualisation et les bases de données. ॥ y a certainement d'autres corrélations importantes à envisager entre les champs du numérique, et des dimensions méthodologiques et conceptuelles supplémentaires se révèleront certainement lors de la mise en œuvre. C'est un ensemble en construction, apportant une grappe de possibilités nouvelles aux historiens, mais aussi d'importantes questions de recherche en sciences de la conception et en informatiqueà prendre en compte. De tels projets offrent des perspectives intéressantes de développement au service de l'histoire des sciences et des techniques, au profit de leur médiation avec le public. Ils nécessitent aussi des collaborations interdisciplinaires auxquelles nous ne sommes pas toujours bien préparés, mais qui sont fructueuses à l'usage. Ils constituent enfin un apport pédagogique incontestable dans les formations universitaires et en écoles d'ingénieurs. 
Ce numéro souhaite dresser un panorama des recherches actuelles et présenter quelques réalisations ou projets remarquables. II devrait s'en dégager une série d'informations et de suggestions pour les lecteurs, également quelques lignes de forces aptes à éclairer la réflexion et à stimuler la recherche à propos de l'usage de ces différents outils.

Une étape semble aujourd'hui franchie et il est permis de penser qu'un premier seuil de maturité est atteint. Cela est tout particulièrement vrai dans la numérisation des images et des textes, comme dans le projet du CNUM, afin de renouveler l'approche documentaire et les pratiques de la recherche, en histoire des sciences et des techniques comme dans tout autre champ historique. La démarche de la base de données et de constitution du portail sur le patrimoine scientifique et technique, en France, est un autre domaine arrivé à sa plénitude. De leur côté, les maquettes numériques devraient apporter des compléments décisifs à la muséographie et au patrimoine dans les années à venir.

L'usage du numérique apparaît finalement comme celui d'un outil de base, parfois de plusieurs successifs, au sein d'un processus d'étude. II nous renvoie aux fondamentaux de la pratique de l'histoire par une patiente investigation des sources, une acquisition des données, puis par l'effort de compréhension et de restitution des faits. En aucun cas il ne remplace les méthodes de l'histoire, de l'archéologie, du patrimoine ou de la muséographie, mais il les complète et les fait évolver, parfois assez radicalement, offrant des possibilités nouvelles de compilation et de mise en scène des connaissances. C'est une démarche de travail souvent très puissante et efficace. Prenons un seul exemple : le relevé manuel au théodolite des cotes d'un bâtiment permettait d'acquérir quelques dizaines de points par façade, mais difficilement plus. Un relevé au théodolite laser permet d'en acquérir des millions en quelques minutes, une fois l'appareil en place, et de reconstituer une image parfaitement rigoureuse des surfaces dont les coordonnées de chaque point sont enregistrées avec une très grande précision. C'est pour cela que les professionnels de ces questions parlent de réalité virtuelle.

L'usage des outils numériques fait évolver les méthodes de travail, parfois radicalement. En retour, il interroge sur les pratiques, voire sur les concepts même de la discipline et sur la manière de les mettre en œuvre. Dans cette lignée d'expérimentations, les questions méthodologiques ouvrent rapidement sur des interrogations plus fondamentales, touchant à la nature même des projets de connaissance ou de valorisation en direction des publics.

\section{Des premiers essais en architecture et en archéologie} aux maquettes numériques animées

La réalisation de maquettes d'étude est fort ancienne, notamment en architecture. N'oublions pas que les modèles réduits de machines sont une partie du projet pédagogique des Lumières, puis de la Révolution française pour le développement de l'éducation technique. Elles touchent alors très directement aux collections, à l'apprentissage d'un savoir-faire, à la diffusion d'une culture technique, puis à des projets muséographiques ambitieux comme ceux du Conservatoire national des arts et métiers, du British Museum ou du Deutsche Museum.

Dans les années 1980, le dessin assisté par ordinateur, puis la possibilité de maquettes numériques tridimensionnelles sont venus renouveler ces problématiques anciennes, en particulier pour les études architecturales et techniques, en offrant une alternative ambitieuse aux pratiques du dessin technique conventionnel, jusqu'à le supplanter dans le monde industriel.

Les maquettes numériques d'architecture et d'archéologie Les deux premiers domaines historiques gagnés par l'usage des nouveaux outils de visualisation sont incontestablement, dès les années 1990, l'archéologie et I'histoire de l'architecture. La possibilité de maquettes tridimensionnelles ou 《 3D ॥ a profondément renouvelé la pratique du dessin de restitution des sites et des monuments. Le projet Maior Eclessia, de l'École nationale des arts et métiers de Cluny, visait à reconstituer virtuellement la grande église abbatiale de l'Ordre clunisien, par un projet alors très novateur de maquette virtuelle 5 . Cette magnifique église fut la plus grande de son temps, après Saint-Pierre de Rome, mais il n'en reste aujourd'hui que quelques éléments architecturaux résiduels. Le projet a lancé en France l'usage de ces nouvelles techniques graphiques et les a popularisées. Depuis, de nombreux programmes de restitutions virtuelles ont été entrepris et menés à bien dans ces domaines. Dans le cadre universitaire français, il faut notamment citer l'équipe AUSONIUS à l'Université de Bordeaux III', en archéologie, ou encore le projet de reconstitution virtuelle de la Rome antique par l'équipe CIREV de l'Université de Caen'.

Les logiciels 3D de réalité virtuelle permettent de restituer des édifices, sous forme de maquettes numériques, à partir de leurs vestiges et en utilisant les données archéologiques et documentaires

5 Le site de Cluny numérique, ENSAM Cluny: www.cluny.eu/

6 /www-ausonius.u-bordeaux3.fr/

7 www.unicaen.fr/services/cireve/rome/ 
disponibles. Ils incluent dans la maquette les dimensions géométriques de l'édifice, en respectant rigoureusement la précision des relevés de terrain. Ceux-ci ont été faits pendant des générations au théodolite et à la règle, accompagnés de calculs manuels puis de transcription sous forme de dessins cotés, de plans et d'élévations à l'échelle. La numérisation des procédés manuels et sa traduction graphique sur un écran, substitut de la planche à dessin, offrait une continuité méthodologique qui a sans doute grandement facilité son emploi dans l'architecture et l'archéologie. Dans ces disciplines, comme pour les mécaniciens, le détour graphique est un intermédiaire essentiel, un support de la pensée et de son expression.

L'outil permet aussi, par un recours approprié aux couleurs et à leur intensité, de distinguer ce qui est certain des hypothèses faites par l'historien ou l'architecte, exactement comme le fait un restaurateur scrupuleux. Cela est toutefois bien plus facile et largement diversifié dans le champ du virtuel, par un nuancier des plus larges permettant par exemple d'indiquer le degré de vraisemblance de ces hypothèses. L'intérêt pédagogique de ces restitutions virtuelles est évident. L'usage des fausses couleurs, des traits indiquant les lignes de forces peuvent donner une grande clarté visuelle aux maquettes, également l'usage du zoom pour des changements d'échelle instantanés, les effets de transparence, la variété possible des points d'observation, etc. ; tout cela sous l'expresse réserve d'un respect scrupuleux des données scientifiques de départ. Les exigences déontologiques de base ne sont pas de nature différente de celles du travail de l'archéologue ou de I'historien de l'architecture. Rappelons que de tels outils ont un usage professionnel en architecture et en urbanisme, comme aide à la conception et à la construction des bâtiments. Ils sont également devenus importants dans le suivi et la restauration des ouvrages d'art et des monuments anciens ${ }^{8}$.

Rapidementcesreprésentations tridimensionnelles (3D) se sont accompagnées d'éléments d'animation permettant des visites virtuelles ou des constructions - déconstructions à but explicatif. La visite virtuelle repose sur le principe de la mobilité de l'œil, repère d'observation, par rapport à un ensemble 3D qui lui reste fixe, avec des effets de zoom, de vue d'ensemble, de perspective cavalière, etc. L'observateur est rendu mobile mais l'objet reste fixe, ce qui correspond bien à une situation de visite

8 Par exemple : Anne Coste, L'architecture gothique, lectures et interprétations d'un modèle, Saint-Etienne, CEF-PU Saint-Étienne, 1997. archéologique ou architecturale. Toutefois, les termes d'un tel parcours, guidé par les clics de la souris, sont d'ordre strictement virtuel, ajoutant au passage une puissance d'accès visuel sans commune mesure avec celle d'une visite réelle du site. Soulignons que la différence du ressenti et du perçu entre ces deux types de reconnaissance d'un lieu est considérable, ce qui distingue bien les deux univers, réel et virtuel, tout en montrant leur très grande complémentarité.

L'apport de la conception assistée par ordinateur Nous abordons la question des maquettes numériques de machines anciennes en lien avec notre expérience personnelle, entamée au début des années 2000 à I'Université de technologie de Belfort - Montbéliard, avec l'aide de Samuel Deniaud du laboratoire $M 3 M^{9}$, puis poursuivie à l'Université de Nantes grâce à l'appui d'Alain Bernard de l'IRCCYN ${ }^{10}$ à l'École centrale de Nantes.

Une spécificité de l'approche de l'historienarchéologue des techniques est la question de la cinématique, c'est-à-dire du compte-rendu du mouvement et des temporalités de l'objet, de la machine ou du processus qu'il étudie. II a tout intérêt à accéder le plus scientifiquement possible à cette activité passée de son objet d'étude, activité pour laquelle il a été construit et utilisé. Ces préoccupations le distingue de l'approche a priori statique des situations d'archéologie architecturale que nous venons d'examiner. Pour cela, la présence de la variable temps doit être introduite dans la maquette elle-même, ce qui implique de pouvoir disposer d'un logiciel graphique simulant un espace à quatre dimensions, les trois de l'espace géométrique classique et celle supplémentaire du temps; un espace dit «3D + †» pour le distinguer du 3D purement géométrique. En d'autres termes, il s'agit de restituer la dynamique d'un processus technique, de l'ajouter aux situations statiques déjà prises en compte.

Il est en effet possible aujourd'hui d'ajouter des animations virtuelles à la $3 \mathrm{D}$ classique à moindre coût, à l'aide de logiciels spécifiques parfaitement capables de créer l'image du mouvement ${ }^{11}$. Toutefois ces outils numériques très souples, largement pratiqués par les agences de communication et les infographistes, restent dans le domaine d'une entière virtualité, c'est-à-dire qu'ils ne dépendent que de l'imagination et de l'habileté de leur utilisateur. IIs ouvrent la voie à des univers totalement imaginaires,

9 Laboratoire de Mécatronique, Méthodes, Modèles et Métiers, UTBM, EA.

10 Institut de recherche en communication et cybernétique de Nantes, UMR CNRS.

11 Les logiciels du type 3D studio-max, Flash, etc. 
comme ceux des jeux vidéo et des films d'animation. La qualité des produits obtenus, en termes de fidélité à la rationalité des faits est alors des plus hypothétiques, et ce n'est généralement pas le but recherché. Elle ne dépend que de la compétence des praticiens et de leurs objectifs, assez peu des contraintes de la réalité archéologique. Les infographistes cherchent au mieux des effets réalistes, mais ils ne traduisent pas scrupuleusement le monde réel. Rien ne garantit la véracité de ce travail d'illustration quand il est censé décrire une machine ayant réellement existé, pas plus que naguère celle d'une représentation par le dessin libre sur papier'2. II s'agit d'une fiction, dans le meilleur des cas d'une évocation réaliste, mais non d'une réalité virtuelle, au sens fort donné aujourd'hui à ce terme par les ingénieurs lorsqu'ils utilisent la conception assistée par ordinateur ou CAO. II y a là une ligne de démarcation essentielle, entre outil ludique et outil scientifique, pas toujours bien comprise et qu'il nous appartient de mettre en évidence, comme entre d'un côté le dessin libre, l'illustration, et de l'autre le dessin technique coté ou le plan d'architecte.

L'outil de la CAO est aujourd'hui utilisé par les professionnels pour concevoir les objets industriels et les machines. II se caractérise par la présence de coordonnées géométriques et temporelles très nombreuses pour définir les dimensions des pièces, les formes et les volumes, les axes et les lignes de mouvements. II intègre rigoureusement les pièces entre elles etilindique immédiatementles contradictions pour non-conformité aux lois mécaniques et physiques qui les régissent. II reproduit les mouvements et leurs règles, tant cinématiques que dynamiques et mécaniques. C'est fondamentalement un outil scientifique de prévision de la réalité géométrique et physique des objets, dans un espace virtuel à quatre dimensions, le fameux « $3 D+\dagger$ » des mécaniciens. Nous l'avons dit, il a remplacé l'anticipation du dessinateur industriel ; il a amplifié et démultiplié l'exigence de ses règles autrefois patiemment apprises à la table à dessin. Cette idée d'appliquer un outil parfaitement rigoureux dans son respect des données matérielles de l'objet nous a semblé très prometteuse pour espérer capter la réalité des machines du passé, dans leur conception et dans leur usage, bientôt dans leur environnement ; une réalité existentielle des objets-machines qui s'arrête brutalement à la fin de leur vie, généralement synonyme d'abandon ou de destruction.

12 Michel Cotte, Florent Laroche, Alain Bernard, « Les outils de réalité virtuelle sont-ils applicables au patrimoine technique et industriel ? "1" Historiens et géographes, Le patrimoine industriel, $2^{e}$ partie, $n^{\circ}$ 401, février 2008, pp. 245-255.
Notre expérience personnelle ne concerne vraiment qu'une case du tableau du chapitre 1, celle justement de rendre compte de la réalité géométrique, physique et technologique des objets anciens. Elle a été de suggérer l'idée d'appliquer les outils de la $\mathrm{CAO}$, et plus largement des outils des sciences pour l'ingénieur à l'analyse des objets de l'histoire des techniques, les machines du passé notamment ${ }^{13}$. L'outil est exigeant et il demande des compétences mais, en contrepartie, il garantit la restitution d'une réalité virtuelle à part entière, d'une grande conformité à l'original en situation de fonctionnement.

Grâce à la compréhension et à l'implication de plusieurs collègues des sciences de la mécanique et de la conception, puis de l'informatique ${ }^{14}$, nous avons pu concrétiser ces idées, montrer leur faisabilité, mettre en évidence leurs apports à la pratique de l'histoire des techniques, puis cerner une approche conceptuelle et méthodologique. A ce jour, ces collaborations ont permis la restitution approfondie d'une petite dizaine de machines ${ }^{15}$. Un travail véritablement pluridisciplinaire nous a permis de réaliser ces projets et d'en percevoir toute la richesse, par des résultats souvent étonnants, notamment dans la connaissance des machines étudiées. Des interrogations et des limites apparaissent aussi, qu'il ne faut pas éluder et qui stimulent la réflexion sur nos pratiques.

Les perspectives de développement sont remarquables ; elles commencent à être bien comprises et convenablement conceptualisées ${ }^{16}$. Elles ont également fait l'objet d'une tentative

13 Nos premiers travaux de recherche ont conduit à deux communications orales, avec Samuel Deniaud : colloque ICOTHEC, Moscou, août 2003 ; atelier ARTEFACT, Vienne, octobre 2003 ; ils sont présentés dans : Michel Cotte, Samuel Deniaud, « Conception assistée par ordinateur et patrimoine, perspectives innovantes ", L'archéologie industrielle en France, 46, juin 2005, pp. 32-38.

14 Outre Samuel Deniaud et Alain Bernard déjà cités mentionnons : Stéphane Sire (UBO Brest), Sébastien Le Loch et Jean-Pierre Guédon (Université de Nantes).

15 Une presse Bliss à Fêches-le-Châtel (Doubs), une machine à vapeur Piguet (Écomusée du Creusot), la machine à noyaux de l'atelier de fonderie (PSA, Sochaux, Doubs), deux machines d'imprimerie (Musée de l'imprimerie, Nantes), la machine à laver le sel (Musée de Batz-sur-Mer, Loire-Atlantique), une pompe à vapeur de marine (École de la marine marchand, Nantes), une restitution dynamique du pont national à Brest (pont tournant à ce jour disparu), un canot à vapeur du XIXe siècle (DCNS Indret, Loire-Atlantique).

16 || s'agit notamment de la thèse de Florent Laroche : "Contribution à la sauvegarde des objets techniques anciens par l'archéologie industrielle avancée ", IRCCyN, École centrale de Nantes, décembre 2007. 


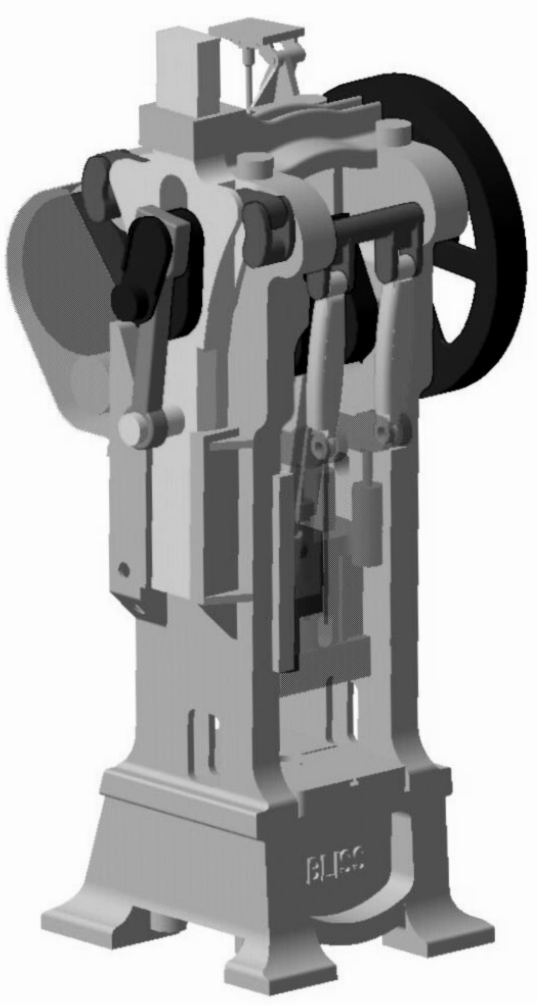

fig. 2 - La première maquette numérique animée réalisée à I'UTBM. II s'agit de l'une des deux presses Bliss, d'origine allemande, commandées par les usines Japy au cours des années 1900 pour leur site de Fesches-le-Châtel (Doubs). Initialement prévues pour des casques de pompiers, elles furent surtout dédiées à la production massive des casques des poilus de la Grande Guerre. Le bâti central de la machine, avec son cœur mécanique, furent sauvés du ferraillage par un poids excédant les capacités de l'engin de levage... Elle fut réhabilitée comme témoignage du patrimoine industriel de la ville à son entrée. (étude technique et numérique coordonnée par Samuel Deniaud).

de structuration par un groupement d'intérêt de recherche, de 2004 à aujourd'hui, par le biais du Centre François Viète et l'Institut de l'Homme et de la Technologie à l'Université de Nantes, de l'IRRCyN à l'École centrale de Nantes, de I'UBO à Brest, formant le pôle Objet, Société et TIC (OSTIC) qui a déjà organisé ou coopéré à la tenue de cinq journées d'études ${ }^{17}$.

\section{La méthode de la maquette numérique ${ }^{18}$}

L'acquisition des données constitue le point de départ : c'est la documentation du sujet que l'historien apprend à

17 Une seule de ces journées d'études a été publiée: $R \& D$ dans l'entreprise : conserver l'instrumentation, pourquoi, comment ?...., Paris, Musée des arts et métiers, 2007.

18 Cette partie fait essentiellement référence aux travaux de Florent Laroche, thèse citée, 2007 et à nos nombreuses discussions lors de sa préparation.

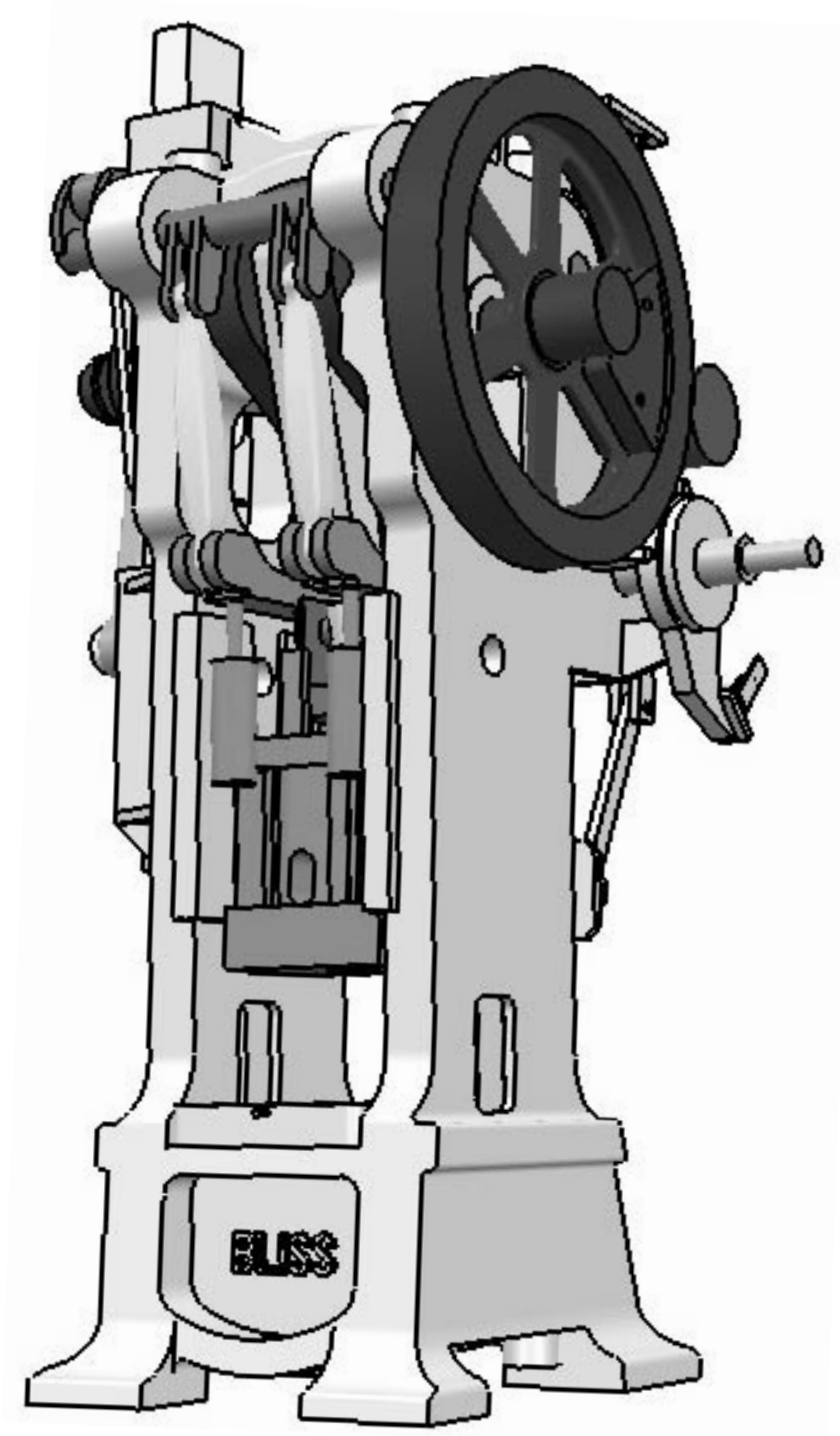

faire de son côté à partir des archives et que le spécialiste du patrimoine pratique par l'inventaire. En termes d'outils, la démarche s'est au fil du temps enrichie de la photo argentique, du film, de la photocopie, de l'enregistrement sonore, puis de la photo et du film numérique, enfin du scan laser 3D pour la réalisation des maquettes.

Cela constitue ce que Florent Laroche dénomme l'état A de son processus d'archéo-histoire avancée, une étape d'analyse systématique des données puis de leur enregistrement numérique. II note avec justesse l'interpénétration du travail de l'historien, du mécanicien-électronicien et de l'informaticien pour y parvenir ; la nécessité aussi d'un ensemble d'acquisitions homogénéisé pour pouvoir être traité et compilé par les logiciels informatiques et dans des environnements compatibles avec les autres étapes du processus.

Ce travail de définition et de normalisation de l'information constitue une transition, une encapsulation des items informationnels qui vise à les rendre 
opérationnels au sein de la programmation elle-même. En d'autres termes, il a fallu transformer les données brutes : textes, images, nuages de points ${ }^{19}$, données cinématiques, etc., en fichiers numériques exploitables par le logiciel de CAO ou par celvi de la gestion de la base de données. Leur intégration progressive construit l'état $B$, une étape purement numérique dont la finalité est une synthèse des données acquises sur l'objet réel. C'est une forme concentrée et organisée de toute l'information, sous deux facettes complémentaires : la maquette numérique et la base de données associée, avec l'ambition prochaine de pouvoir circuler aisément de l'une à l'autre. L'un des objectifs de ces travaux est d'ajouter à la visualisation dynamique de l'objet un hypertexte informatif évolvé. L'état B achevé constitue alors un dossier d'œuvre archéo-historique. Une nouvelle pièce d'archive est constituée pour le présent comme pour le futur, bientôt consultable librement à distance pour la documentation, la recherche, la pédagogie, etc., du moins nous l'espérons ${ }^{20}$. Une des conditions est toutefois de savoir organiser et institutionnaliser une archivistique durable de cette information numérique, de prévoir une norme pour rendre les différentes expérimentations compatibles entre elles, donc cumulatives et comparables. L'état $B$ est un objet scientifique en soi, un noyau dur de la connaissance, un objet virtuel pouvant être enrichi, évalué, mis en perspective avec d'autres similaires. II est alors transmissible et restituable en termes compréhensibles et rationnels. C'est une réalité condensée du passé par la puissance du virtuel et de l'information numérique.

À partir de là, l'utilisation du dossier d'œuvre numérique est largement ouvert, vers des états $C, C$ ', $C^{\prime \prime}$, etc., en fonction d'un cahier des charges fourni par un projet d'utilisation : complément numérique à la muséographie, programme pédagogique, recherches professionnelles de conception - innovation, etc. Citons un exemple d'utilisation aboutie d'une maquette numérique : la machine à laver le sel de Batz-sur-Mer était dans un état de ruine avancé et sans restauration possible ; sa maquette numérique a finalement conduit les responsables du Musée des marais salants à une reconstruction, autorisée par le dossier d'œuvre numérique très complet21 . En termes techniques, il s'agit d'une rétro-conception conduisant à un fac-similé très

19 On appelle ainsi le résultat des relevés spatiaux numérisés de l'objet, rappelant qu'il s'agit de coordonnées de points dans un espace géométrique.

20 La dimension d'accès internet fait partie intégrante du projet OSTIC ; toutefois, pour des raisons tant logistiques qu'informatiques, il n'a pu être donné suite à ce projet de site du patrimoine numérique pour l'instant.

21 F. Laroche, thèse citée, 2007. fidèle de la machine originelle, dans un état neuf !

Les techniques de maquettes et les problématiques qui leur sont associées sont elles-mêmes en pleine évolution, par les progrès de la saisie numérique en amont, par les techniques de vision stéréoscopique en aval, l'introduction des environnements, les recherches ergonomiques sur les effets de préhension et le retour d'effort, etc. Ce sont des sujets en cours d'expérimentation, tendant à fournir une réalité augmentée, à côté et en prolongement de l'objet muséographique ou archéologique lui-même. L'association de bases de données à des maquettes numériques ou à de la cartographie historique sont des sujets plus ouverts, en termes de recherche comme de perspectives, mais on en attend beaucoup, en particulier pour l'approfondissement du concept d'interactivité comme pour celvi de la capitalisation des connaissances du passé dans des formes accessibles et transmissibles aux générations futures.

\section{Les temporalités techniques et industrielles, le patrimoine et la CAO}

En prolongement du panorama que nous venons de parcourir sur les usages du numérique en général et de la CAO en particulier, arrêtons-nous un instant sur cette question : qu'est-ce qui caractérise vraiment la notion de patrimoine technique et industriel ? Pour répondre en quelques mots : c'est à notre avis la conservation de la connaissance d'un processus technique visant à produire un objet ou à effectuer une tâche matérielle ${ }^{22}$. Celui-ci est caractérisé par l'utilisation rationnelle d'un ou d'une série d'artefacts par l'Homme, dans un espace - temps donné et au sein d'un système sociotechnique ${ }^{23}$. Ce processus s'inscrit dans plusieurs dynamiques temporelles simultanées, ressortissant d'ordres différents : celle de la machine et de son environnement matériel, celle de l'homme au travail, ou encore les rythmes de la production, des usages, de l'entretien, de l'innovation, etc. ${ }^{24}$.

Plus largement, tout élément technologique s'inscrit dans des repères spatio-temporels dont l'histoire et le patrimoine se doivent de rendre compte. En d'autres

22 Nous laissons de côté les techniques organisationnelles, corporelles ou de service qui sortent de notre propos.

23 Michel Cotte, « La génétique technique a-t-elle un avenir comme méthode de l'histoire des techniques ? ", journées d'études de la SFHST, Lille, 23-25 mai 2007, actes à paraître. 24 Lewis Mumford a sans doute été l'un des premiers à souligner l'importance de la compréhension des phénomènes temporels dans la structuration de la pensée rationnelle occidentale ; voir Technique et civilisation, Paris, Sevil, 1950 ; 1 ère éd. en anglais, 1946. 
termes, la nature profonde du patrimoine technique et industriel est d'ordre dynamique : c'est une action, une transformation, un changement et non un objet figé ou une image instantanée. Bien entendu, avoir une photo ou un plan est mieux que rien, garder la vielle machine est encore mieux ; mais ce n'est pas satisfaisant. Une machine conservée dans un état statique peut assez rapidement devenir une énigme, même dans un musée, un bloc inerte en grande partie dépouillé de ses significations, une masse devenue silencieuse. Toute la difficulté est bien là, et la négliger peut conduire à des contresens sur la nature même de l'héritage technique et industriel que nous sommes en train de recevoir des générations antérieures.

L'historien, l'archéologue connaissent bien ces difficultés, et ils savent que la qualité de leur travail de documentation et d'analyse des sources autour des objets recueillis ne suffit pas. II faut ensuite traduire convenablement l'ensemble des faits en un récit cohérent ; c'est-à-dire les décrire logiquement, montrer leurs corrélations, les comparer, les installer dans un contexte, dans un système sociotechnique, comprendre les enjeux et les environnements socioéconomiques, etc. ; bref, problématiser son étude et avancer des hypothèses pour redonner sens et intelligibilité au passé. Ce n'est pas très différent pour le patrimoine où les faits sont bien là, mais où la question d'une «écriture » à part entière du patrimoine reste balbutiante dès qu'il s'agit de rendre compte, au-delà du discours ${ }^{25}$, de la dynamique des processus techniques et industriels. Les efforts méritoires de présentations de pratiques professionnelles par les "muséographies vivantes " en restent presque toujours à des stades artisanaux, tant pour des raisons de coût, de simplicité d'explication, d'effet visuel que de durée moyenne d'attention des visiteurs. L'autre versant est la projection de films, quand ils ont pu être réalisés avant l'arrêt des ateliers, également les produits multimédias classiques faits de montages à base de photos, de documents, de témoignages et de schémas explicatifs. Ce n'est donc pas si simple de rendre compte des différentes temporalités de la vie passée des objets, surtout lorsqu'il s'agit de machines complexes, a fortiori pour des ensembles. Les maquettes numériques de type CAO doivent pouvoir apporter une aide importante à la réalisation de cet objectif, notamment pour la compréhension technique dynamique de l'objet-machine. Dans

25 Celui-ci, par la parole du guide ou l'ouvrage historique peut bien entendu être de grande qualité et contribuer de manière décisive à l'intelligence d'un lieu ou d'un objet; mais il s'agit ici de franchir une nouvelle étape dans le compte rendu de la réalité historique. cette optique, l'analyse des temporalités dans lequel il s'inscrit devient une étape préalable au passage vers le virtuel.

Temps et rythmes propres aux machines

Le premier temps de la technique est dans le fonctionnement même de la machine. C'est tout d'abord la durée élémentaire de son cycle d'action, généralement répétitif ${ }^{26}$. II s'agit par exemple des tours par minute d'un moteur, du va-et-vient d'un mécanisme. Plus largement, c'est la période d'un phénomène matériel ou énergétique, une grandeur définie par les physiciens, en secondes, et dont l'inverse mathématique s'appelle la fréquence, en hertz. La technologie industrielle est souvent basée sur de tels phénomènes répétitifs, dont bien entendu les caractéristiques peuvent varier, accélérer ou décélérer, se modifier en amplitude, s'arrêter pour ensuite repartir. La maîtrise du rapport au temps des objets techniques a fasciné diverses communautés au cours des âges: les constructeurs d'automates au XVIII siècle, les fabricants de moteurs à vapeur puis à combustion interne, les premiers concepteurs d'ordinateurs à la fin des années 1940, etc. N'oublions pas qu'il s'agit de la raison d'être de l'industrie horlogère, paradigme du temps apprivoisé par la technique humaine ${ }^{27}$.

Dans cette réflexion sur les fondements du patrimoine technique, on objectera peut-être une trop grande proximité avec le monde des mécaniciens. C'est vrai, et l'outil de la CAO vient d'eux, destiné à la conception des machines. Toutefois, les phénomènes périodiques en technologie sont bien plus larges que cela : les oscillations et les vibrations des structures, le balayage électronique des écrans vidéo, le multiplexage de l'information, les horloges à quartz au cœur du fonctionnement des ordinateurs, la transmission électromagnétique des données, etc. Bref, les périodes en jeu dans le fonctionnement interne des objets techniques peuvent aller de quelques minutes à des micro ou nanosecondes.

Au passage, il est particulièrement difficile d'envisager la représentation des phénomènes les plus rapides en direction du grand public, en particulier ceux de l'électronique, sauf à réaliser des animations virtuelles à vitesse humaine, simple simulacre visuel d'une réalité imperceptible. Nous sommes-là

26 Bien entendu, les machines à commandes numériques peuvent obéir à des cycles algorithmiques complexes et en changer au gré des besoins productifs.

27 David S. Landes, L'heure qu'il est ; les horloges, la mesure du temps et la formation du monde moderne, Paris, Gallimard, 1987 ; 1 ère éd. en anglais, 1983. 
devant la question de la technologie boite noire, qui semble l'une des caractéristiques d'évolution de notre époque, et que nous ne pouvons ignorer. Une distance grandissante se crée entre les usagers et la réalité non visible des phénomènes physiques en jeu, que seuls l'éducation et la culture scientifique peuvent tenter de combler. Notre démarche ambitionne bien entendu de soutenir ces efforts.

\section{De la temporalité des processus}

aux temporalités humaines

Le second rapport au temps est la durée des processus : le temps pris pour la fabrication du produit par la machine, l'atelier ou l'usine. Par exemple : le passage des matériaux dans concasseur à gravier s'exécute en 2 minutes; il fallait 200 heures pour produire la Ford T en 1929 ; l'algorithme est exécuté en 150 millisecondes, etc. Nous touchons-là aux questions de productivité, hier comme aujourd'hui au cœur des enjeux industriels. Le lien s'établit avec les notions de flux et de circulation des matières, des produits, des fluides, de l'énergie, des informations, etc., dont les échelles comme les supports structurels peuvent être extrêmement variables, les temporalités propres aussi. Dans le défi de la conservation patrimoniale, la complexité de l'espace rejoint celle du temps.

La notion de temps d'un processus s'étend facilement à celle voisine, mais distincte, de durée d'usage : le four doit fonctionner 30 minutes à $180^{\circ} \mathrm{C}$ pour la tarte aux pommes, le transport TGV jusqu'à Lyon demande 1 heure 55 minutes, les systèmes de commandes hydrauliques doivent être révisés après 300 heures de vol, etc. Ces durées sont des variables adaptables, mais elles constituent des éléments prévisionnels indispensables au bon usage de la technique. Elles sont commandées par l'homme, par sa planification, mais aussi et de plus en plus par des programmes automatiques spécialisé ${ }^{28}$. Dans notre inventaire des temporalités, il apparaît là un nouveau rapport au temps, celui à l'interface de l'hommeusager ou del'homme-producteur avec la machine. En d'autres termes, la temporalité humaine se confronte à celle propre aux objets techniques, dans ce que l'on peut appeler le temps ergonomique, plus largement le temps du travail ou de l'usage. Sujet immense, bien entendu, que le temps du travail : l'Homme y confronte des données simultanément bio-morphologiques, sociales et techniques où les temporalités jouent un rôle essentiel : horaires, pointeuse, durée normalisée des tâches, rendement, fatigue, répétitivité,

28 Bruno Jacomy, L'âge du plip, chroniques de l'innovation technique, Paris, Seuil, 2002. optimisation des gestes, alimentation, sommeil, arc réflexe musculaire, durée de la perception rétinienne, repos, maladies professionnelles, etc. Nous ne faisons ici que mentionner des facteurs directement associés aux temporalités du travail humain, constituant des éléments importants du patrimoine technique et industriel. II en va de même pour l'évocation de la relation ergonomique, comprise dans le sens large des relations que l'homme a entretenu avec ses machines tout au long de l'histoire, jusque dans ses représentation, jusqu'à penser qu'elles étaient faites à son image, ou l'inverse ${ }^{29} \ldots$

Les sciences de la production, dont le taylorisme est l'une des racines mais il y en a bien d'autres, ne cessent aussi de confronter les temporalités : celle de la parcellisation optimisée et distribuée des tâches entre l'homme et la machine, celle de l'intelligence d'ensemble des processus et de leur contrôle, celle de l'économie de la production, etc. ${ }^{30}$. Les temporalités de l'homme et de la machine ne sont pas de même nature, mais elles sont à accorder pour un usage efficace. Cela n'est pas spontané ; la relation ergonomique d'usage de la machine doit être soigneusement pensée, sujet par excellence d'innovations incrémentales. Les capacités biologiques de l'homme sont stables au cours des âges (vision, réflexes, mouvements) ; elles sont toutefois améliorées par l'apprentissage, optimisées par une bonne ergonomie des machines et des ateliers, une bonne gestion des tâches, voire poussées à leurs limites par le stress quotidien de la productivité. En dehors du champ industriel, l'utilisation du changement de vitesse du VTT offre un excellent exemple de l'adaptation ergonomique permanente des rythmes humains à ceux de la machine, en fonction de la tâche à accomplir.

D'autre part, l'évolution de la temporalité des machines s'effectue vers I'ultrarapide, notamment via les technologies électroniques et électromagnétiques. Il en va de même à propos des échelles de plus en plus microscopiques des composants fondamentaux, comme de leur complexité intrinsèque. Nous retrouvons l'extrême difficulté à rendre compte de manière sensible des espaces - temps de ces nouvelles technologies, alors qu'elles s'éloignent de plus en plus des échelles humaines, à l'image des nanotechnologies ${ }^{31}$.

29 Siegfried Giedion, La mécanisation au pouvoir, Paris, DenoëlGonthier, 1980 ; David E. Nye, American technological sublime, Cambridge Mass. USA, MIT Press, 1994.

30 Herbert A. Simon, Les sciences de l'artificiel, Paris Gallimard, 2004 ; 1 ère éd. en anglais, 1996.

31 Cette difficulté pédagogique est partagée avec les sciences de l'infiniment petit et de l'infiniment grand. 
Les temps de l'innovation et de l'obsolescence L'objet lui-même a une histoire qui s'inscrit dans la durée : sa conception, sa mise en fabrication, son achat par un usager, I'histoire de son utilisation, de ses réutilisations éventuelles, de son rejet enfin, ou... de son éventuelle revalorisation comme objet de patrimoine ${ }^{32}$.

Comme pour le temps du travail, nous sommes sur un terrain pour l'instant un peu loin des techniques numériques classiques. Toutefois, des scénarios de petits films ou de récits sur la vie ou sur l'usage des objets sont parfaitement envisageables, sans avoir d'ailleurs forcément recours au numérique. Les publicités sur les arts ménagers, dans les années 19501960, en donnent une version optimiste et naïve à propos des usages, une image du progrès dont le parfait contrepoint humoristique est le film Mon oncle, de Jacques Tati (1956). Il s'agit-là de la mise en scène d'un temps anthropologique de l'objet, y compris de ses représentations ${ }^{33}$, qui fournit déjà des sujets d'exposition et des possibilités muséographiques intéressantes.

Un autre rythme de l'histoire technique et industrielle doit être évoqué, celui du renouvellement plus ou moins rapide des installations de production, plus largement des technologies elles-mêmes. Elles sont en règle générale l'objet d'innovations, de modifications, parfois d'abandon. "I y a là des temporalités conditionnées par divers paramètres : le vieillissement et l'obsolescence technique, le rôle de la concurrence et l'état du marché économique, l'innovation, enfin les choix stratégiques des entrepreneurs ${ }^{34}$. Le rythme propre du renouvellement technologique peut varier considérablement d'une branche industrielle à une autre. La loi de Moore de l'industrie électronique suggère un doublement des performances techniques tous les dix-huit mois environ, ce qui fait que l'étude d'un circuit imprimé pour un constructeur automobile, dont le temps de recherche et développement est d'environ trois ans, rend ce circuit imprimé obsolète le jour même de sa mise sur le marché. En termes d'histoire des techniques, il s'agit de comprendre une filière technique dans sa complexité et sur la durée beaucoup plus qu'en objet isolé.

Par extension, nous arrivons à la temporalité de la patrimonialisation des objets techniques comme

32 Michel Cotte, «Contribution à une réflexion sur la nature de l'histoire des techniques: une approche systémique de I'histoire d'un objet ", Poitiers 20 mai 2004, colloque de la SFHST, [communication non publiée].

33 Thierry Bonnot, La vie des objets, Paris, éd. de la MSH, 2002. 34 Patrice Flichy, L'innovation technique, Paris, éd. La Découverte, 1995. de leurs lieux de production ou d'usage, quand un concours de circonstances a permis leur conservation. Ce n'est en rien un temps linéaire et paisible où l'accumulation des ans donne ipso facto noblesse et valeur mais, au contraire, un temps souvent de crise lié à l'abandon, à la délocalisation, à la reconversion, à l'apparition de friches industrielles, etc. II y a le paradoxe d'une reconnaissance naissant par la brusque dévalorisation d'une vocation technique locale, avec ses conséquences économiques et sociales. Un site en cours de patrimonialisation essaie de conserver quelque chose, au moment même où l'existence de cette chose est remise en cause, du moins en ce lieu et dans cette communauté humainelà. L'objet, sa technique ne disparaissent pas pour autant; ils reprennent vie ailleurs; ils s'inscrivent dans des dynamiques géoéconomiques et capitalistes plus anciennes qu'on ne le pense généralement, plus largement dans un mouvement de circulation et d'adaptation des idées techniques ${ }^{35}$. L'industrie est faite de migrations incessantes dans l'espace et dans le temps, d'appropriations et de réappropriations, alors que le patrimoine semble vouloir retenir et figer le temps qui passe, tout comme ses lieux. Le patrimoine ne peut en rien être réduit à une nostalgie de la conservation, sinon il ne survivra pas au simple changement de génération, et de nombreux acteurs du patrimoine industriel sont pleinement conscients de cet écueil.

Nous touchons bien là aux difficultés épistémologiques du concept de patrimoine technique et industriel. La principale concerne la dimension statique du patrimoine, par essence bien immobilier, confrontée à la mobilité congénitale des techniques et de l'industrie dans les différentes temporalités que nous venons d'esquisser. II arrive que le concept de patrimoine industriel soit réduit à ses vestiges architecturaux, plus robustes en termes de survie que les machines elles-mêmes, plus conforme aussi à sa définition juridique ${ }^{36}$. Par ailleurs, la tradition générale du patrimoine, notamment en France, se décline en termes de monuments, d'ensemble architecturés et urbains. Tout cela risque

35 David S. Landes, Richesse et pauvreté des nations, Paris, Albin Michel, 2000 ; 1 ère éd. en anglais, 1998 ; Michel Cotte, De l'espionnage industriel à la veille technologique, BelfortBesançon, UTBM-PU de Franche-Comté, 2005.

36 Par exemple, la Convention du patrimoine mondial s'applique à des biens immobiliers et la dimension mobilière en est a priori exclue. En clair, un patrimoine par exemple ferroviaire candidat à ce label international est constitué par la ligne ferroviaire, ses infrastructures, ses bâtiments, éventuellement par ses paysages, mais pas par son matériel roulant. 
d'être purement statique, pétrifié en quelque sorte, cristallisé disent les puristes de la conservation.

Une seconde difficulté porte sur la coupure passé - présent, parfois attachée aux perceptions par le public de l'acte patrimonial ou muséographique. Un patrimoine technique et industriel qui n'inscrirait pas son projet de conservation dans une valorisation pleinement liée au présent a sans doute peu de chances d'aboutir, peu de chances de dépasser l'effort volontariste qui l'a généré à un moment donné. C'est bien entendu la question beaucoup plus globale des relations qu'une société entretient avec ses racines, ses lieux de mémoire; et elle est souvent plus ardue pour le patrimoine industriel que dans d'autres domaines comme l'architecture ou l'art. Elle mérite d'être posée pour elle-même, pour prendre conscience des faux-fuyants que représente la dérive vers une simple approche architecturale ou paysagère des sites de l'industrie passée, ou encore des programmes de réhabilitation qui, souvent, ignorent et oublient le patrimoine industriel ou bien n'en font qu'un vague prétexte.

Ce n'est pas pour nous une opposition entre patrimoine et architecture, loin s'en faut, mais un appel à dire honnêtement ce que l'on fait, et ne pas faire passer l'un pour l'autre. Le patrimoine technique et industriel ne se réduit pas au patrimoine de son architecture, pas seulement par incomplétude mais parce que ses attributs sont de nature différente. Inversement, les processus techniques représentés par les machines et les hommes qui les utilisèrent s'inscrivent toujours dans des lieux, des espaces bâtis, des environnements géographiques avec lesquels ils entretiennent de nombreuses relations.

Les temps de la conception et de la rétro-conception Notons enfin que le travail de l'ingénieur en conception, plus largement du designer, est une projection sur l'axe des temps, vers le futur, alors que celui de l'historien l'est aussi, mais vers le passé, c'est-à-dire dans l'autre sens de l'axe ${ }^{37}$. La démarche de concepteur est une capacité à penser rationnellement un ensemble technologique dans un avenir plus ou moins proche, à prévoir les conditions de sa production, de son usage et de sa réussite commerciale. Tous les projets, toutes les inventions ne

37 Pour incongru que puisse paraître ce rapprochement, il nous a semblé un élément culturel important ayant favorisé le « travailler ensemble ॥ avec non seulement les collègues mécaniciens, mais avec les designers et les architectes, en particulier Erwin van Handenhoven à Belfort, l'Ecole de design à Nantes. Nous avons notamment esquissé ensemble le concept de «boucle temporelle de la conception », non publié. réussissent pas, loin s'en faut. La CAO est précisément un outil d'aide à cette projection temporelle, à l'énoncé des conditions scientifiques dans lesquelles elle peut prendre place et se réaliser. C'est la préparation du réel par le virtuel numérique, espace techniquement renouvelé de l'exercice ancien de la pensée et du travail de conception.

La démarche CAO appliquée au patrimoine technique et industriel est une démarche simplement inversée sur l'axe des temps, mais utilisant le même outil et les mêmes règles du jeu. Du point de vue de l'ingénieur, il s'agit d'une rétro-conception, d'un passage cette fois du réel vers le virtuel. Inscrit dans un espace virtuel, l'axe des temps peut fonctionner dans les deux sens ; il n'est pas soumis à l'interdit de l'irréversibilité de l'écoulement du temps du monde réel. De ce point de vue, l'outil est neutre, et il peut aussi bien servir à préparer le futur qu'à comprendre et décrire le passé. II apporte une démarche de rétro-conception et de capitalisation des connaissances qui, partant des données d'un passé réel, le transforme en données numérisées aptes à fournir une maquette de référence et, plus largement, un dossier d'œuvre numérique traductible en actions muséographiques ou patrimoniales, en données transmissibles au futur. Enfin, la CAO semble une voie raisonnable de compilation et de restitution des principales temporalités de la machine et de l'industrie, c'est-à-dire pour un archivage à caractère patrimonial conservant les données dynamiques. On nous objectera que seules les plus basiques peuvent être fidèlement reproduites aujourd'hui. Précisément, les efforts de la recherche portent sur des approches numériques d'ensembles de machines, de leurs flux, la restitution de processus complets, la prise en compte dans l'espace virtuel des relations homme machine, des environnements. Bref, c'est un outil en devenir, mais dont le rapport fondamental au temps permet d'espérer une contribution décisive pour une avancée du patrimoine technique et industriel. Conçu pour prévoir le futur des objets, il est au fond des plus logique de découvrir qu'il est tout prêt pour nous aider à rendre compte de leur passé.

\section{Conclusion}

Dans cet article, nous avons voulu esquisser les champs possibles d'application des outils numériques dans l'histoire des techniques, ensuite présenter notre expérience personnelle dans le domaine spécifique des maquettes numériques appliquées au patrimoine technique et industriel, enfin ouvrir une réflexion sur les temporalités induites par l'usage de la CAO dans de telles recherches.

Nous ne reviendrons pas sur la puissance des outils, 
les capacités considérables de développement que l'on peut en attendre dans les années à venir, ni même sur la déontologie indispensable à leur utilisation dans des études historiques et des projets de patrimoine. Quelques points méritent cependant que l'on en rappelle les termes, car le sujet est en devenir et il reste très ouvert.

Même si nous avons esquissé ici un bilan, ce n'est ni un travail abouti ni une réflexion achevée. Sur beaucoup de points la réflexion ne fait même que commencer. Plusieurs éléments s'imposent cependant à nous avec une certaine force. Le premier, comme un préalable, est qu'il ne s'agit pas de remplacer le réel par le virtuel, comme l'ont parfois craint certains acteurs de la muséographie ou du patrimoine, ou d'opposer les champs de l'histoire et du patrimoine avec les techniques numériques, mais de les rendre complémentaires, de mettre la puissance des secondes au service des premiers.

En pratique, l'usage du numérique est d'abord une expérimentation, une exploration de nouvelles méthodes de travail, pour le moins peu familières à I'historien. Mais il peut devenir, parfois très rapidement, un outil indispensable, un média incontournable de notre travail tellement ses avantages sont importants comparés aux savoir-faire antérieurs. II faut avoir conscience de cette rupture dans les usages et garder un esprit attentif aux questions que peuvent suggérer les outils numériques, au-delà et en prolongement de leurs apports pratiques. Par exemple, le traitement dynamique des objets apporté par la CAO interpelle sur la nature même du patrimoine technique et industriel, rappelant l'importance centrale du processus tant technique que social associé à l'usage de la machine, à l'atelier de production.

Dans le cas des maquettes numériques, il s'agit à l'évidence d'un travail interdisciplinaire, d'au moins deux pôles dans un premier temps : historien et mécanicien, de trois et peut être plus ensuite, tant l'apport des informaticiens sous toutes leurs formes est indispensable, notamment lorsque l'on cherche un usage croisé de plusieurs outils. II faut une forme de modestie, d'écoute, d'attention au sens des mots et à leur usage chez chacun. Dans cet article, nous n'avons pas hésité à utiliser quelques termes clés de la conception mécanique, au risque de surprendre I'historien ou le muséographe, tant ils s'imposent dans la pratique du projet interdisciplinaire. Par exemple, la question de l'authenticité, un terme et un concept issu du patrimoine, n'est absolument pas perçue de la même manière par les deux communautés. Pour les sciences de la conception, une maquette virtuelle aboutie contient plus de connaissances que la somme des éléments archéologiques et documentaires qui lui ont donné naissance; elle a retrouvé ses caractéristiques techniques majeures, notamment sa dynamique fonctionnelle; elle est devenue une réalité virtuelle, et si elle comporte des éléments d'environnement et une documentation numérique associée, elle devient carrément une réalité augmentée. C'est un ensemble bien plus riche en informations et bien plus représentatif de la période de vie de l'objet, donc plus authentique que ses vestiges inanimés et partiels!

L'usage des outils numériques en histoire des techniques est tout d'abord de vivre une forme d'insécurité, au-delà des mots et du regard porté par plusieurs communautés sur les objets du passé. Cet usage interroge en permanence sur la manière de travailler, sur la méthodologie d'étude de l'objet technique, sur le sens des mots et des concepts de chacun. II impose une comparaison critique avec les méthodes de l'histoire et de l'archéologie, montrant toutefois des parallèles importants dont l'expression et les modalités diffèrent cependant. Une forme de rigueur étrangère à nos habitudes s'impose pour en comprendre les termes, une rigueur toute technique et professionnelle : on commence par le mètre à ruban et le pied à coulisse pour recueillir de l'information, ou par une saisie numérique et des nuages de points dans un fichier informatique; puis par une analyse fonctionnelle de l'objet, une indexation normalisée des données pour pouvoir les traiter par un logiciel approprié, etc. Bien entendu, il n'est pas demandé à I'historien de faire tout cela par lui-même, mais de collaborer avec d'autres pour y parvenir et en comprendre les enjeux. II apporte, lui, son expérience des archives, son sens du contexte historique, son habitude del'environnementsocio-économique, etc. La coopération ne peut manquer d'être fructueuse et nous l'avons vécue comme telle ${ }^{38}$.

Quelques remarques complémentairess'imposent encore, à propos des limites que paraît porter en ellemême la démarche : il semble ne s'agir que d'un détour sophistiqué vers une restitution purement internaliste des objets techniques. Alors est-ce bien la peine de se donner tout ce mal ? Est-ce bien la peine de passer autant de temps à restituer et synthétiser tous ces détails ? C'est là qu'intervient, pour nous, l'évidence d'un changement profond de système sociotechnique, en train de dévaloriser et de faire disparaître une masse considérable non seulement

38 Nous faisons référence aux stages croisés, organisés dans le cadre OSTIC à Nantes pour l'étude d'un objet-machine, accueillant des étudiants et des élèves issus de différentes UFR, IUT et écoles d'ingénieurs. 
d'objets etdemachines, mais toutsimplementunsavoir et un savoir-faire technique gigantesque accumulé par les générations précédentes. Vu comme un ensemble appartenant à la collectivité humaine, comme le patrimoine de générations d'ouvriers, de techniciens, d'ingénieurs et d'entrepreneurs, c'est une responsabilité considérable. Que faire? L'approche numérique n'est pas brusquement la panacée universelle en réponse à cette immense question, mais c'est un outil important, issu très directement des milieux professionnels. II est en outre capable de fournir des données directement lisibles dans le nouveau système sociotechnique en train de se mettre en place ; c'est-à-dire des informations a priori compréhensibles par les générations à venir, qui auront sans doute bien du mal à interpréter les machines sauvegardées dans les réserves des musées ou même simplement les plans techniques anciens dans les archives.

Sur ce point, nous sommes frappé par le fait que, très souvent, les plans de machines sont les premiers documents à disparaître des ateliers ou des bureaux, probablement pour de simples raisons de stockage car ils sont encombrants à conserver. Par ailleurs, en termes pratiques, des dizaines, voire des centaines de plans et de dessins techniques sont associés à la création, à la construction, à l'entretien et à l'usage d'une machine-outili39. En l'absence de la machine elle-même, et parfois même en sa

39 Nous pensons ici à l'étude Utвм de la machine à noyau de la fonderie de Sochaux pour la fabrication des blocs moteurs juste avant son démontage. présence une fois qu'elle est arrêtée et que son personnel est dispersé, il est souvent difficile de les comprendre et de les interpréter vraiment. Si une synthèse cohérente et rigoureuse, comme la permet une compilation CAO des données, n'est pas faite assez rapidement, nous sommes malheureusement convaincus de la disparition rapide de ce savoir ${ }^{40}$. C'est pourquoi les outils d'aujourd'hui des sciences pour l'ingénieur peuvent permettre une capitalisation des connaissances du passé transmissible aux générations futures. Nous sommes par ailleurs bien conscient qu'un tel travail d'inventaire n'est pas véritablement engagé dans le contexte actuel, sauf si l'entreprise a déjà procédé par elle-même à ce type d'archivage numérique, ce qui est le cas des installations industrielles récentes ou d'entreprises soucieuses de leur patrimoine technique. Ou bien, il faudrait de puissants programmes publics consacrés à l'inventaire et à la conservation de ce patrimoine technique et industriel sous forme numérique, dont il n'existe pour l'instant même pas l'esquisse. Toutefois, cette question a été abordée avec succès pour le patrimoine scientifique des universités, démontrant d'intéressantes perspectives (voir l'article de Yves Thomas et Catherine Cuenca dans ce numéro).

40 Le premier exemple que nous avons eu la chance de connaître, et qui est à l'origine de notre démarche pour l'utilisation de la CAO dans l'histoire des techniques, est la rencontre d'un ingénieur de l'aciérie de Linz (Autriche) en charge de l'installation d'un nouveau train de laminoir et ayant réalisé une compilation CAO de l'ancien au moment de son démontage. 